\title{
A First Search for Prompt Radio Emission from a Gravitational-wave Event
}

\author{
Thomas A. Callister ${ }^{1}\left(\mathbb{0}\right.$, Marin M. Anderson ${ }^{2}$, Gregg Hallinan ${ }^{2}$, Larry R. D’addario ${ }^{2}$, Jayce Dowell ${ }^{3}\left(\mathbb{0}\right.$, Namir E. Kassim $^{4}$ (1), \\ T. Joseph W. Lazio ${ }^{5}$, Danny C. Price ${ }^{6,7}$ (D), and Frank K. Schinzel ${ }^{3,8}$ (i) \\ ${ }^{1}$ LIGO Laboratory, California Institute of Technology, 1200 East California Boulevard, Pasadena, CA 91125 USA; thomas.a.callister@gmail.com \\ ${ }^{2}$ California Institute of Technology, 1200 East California Boulevard, Pasasdena, CA 91125 USA \\ ${ }^{3}$ Department of Physics and Astronomy, University of New Mexico, Albuquerque, NM 87131 USA \\ ${ }^{4}$ Naval Research Laboratory, Washington, DC 20375, USA \\ 5 Jet Propulsion Laboratory, California Institute of Technology, 4800 Oak Grove Drive, Pasadena, CA 91109, USA \\ ${ }^{6}$ Department of Astronomy, 501 Campbell Hall \#3411, University of California Berkeley, Berkeley CA 94720, USA \\ 7 Centre for Astrophysics \& Supercomputing, Swinburne University of Technology, Hawthorn, VIC 3122, Australia \\ ${ }^{8}$ National Radio Astronomy Observatory, P.O. Box O, Socorro, NM 87801, USA \\ Received 2019 March 15; revised 2019 May 8; accepted 2019 May 16; published 2019 June 4
}

\begin{abstract}
Multimessenger observations of the binary neutron star merger GW170817 have enabled the discovery of a diverse array of electromagnetic counterparts to compact binary mergers, including an unambiguous kilonova, a short gamma-ray burst, and a late-time radio jet. Beyond these counterparts, compact binary mergers are additionally predicted to be accompanied by prompt low-frequency radio emission. The successful observation of a prompt radio counterpart would be immensely valuable, but is made difficult by the short delay between the gravitationalwave and prompt electromagnetic signals, as well as by the poor localization of gravitational-wave sources. Here, we present the first search for prompt radio emission accompanying a gravitational-wave event, targeting the binary black hole merger GW170104 detected by the Advanced Laser Interferometer Gravitational-Wave Observatory (LIGO) and Virgo during their second (O2) observing run. Using the Owens Valley Radio Observatory Long Wavelength Array, we search a $\sim 900 \mathrm{deg}^{2}$ region for transient radio emission within approximately one hour of GW170104, obtaining an upper limit of $2.5 \times 10^{41} \mathrm{erg} \mathrm{s}^{-1}$ on its equivalent isotropic luminosity between 27 and $84 \mathrm{MHz}$. We additionally discuss plans to target binary neutron star mergers in Advanced LIGO and Virgo's upcoming O3 observing run.
\end{abstract}

Key words: gravitational waves - radiation mechanisms: non-thermal - stars: black holes

\section{Introduction}

The detection of the binary neutron star merger GW170817 has heralded the era of joint electromagnetic and gravitationalwave astronomy (Abbott et al. 2017a, 2017b; Coulter et al. 2017; Goldstein et al. 2017; Hallinan et al. 2017; Troja et al. 2017). Observed in virtually every electromagnetic band, this event yielded an extraordinary amount of information, including measurements of nucleosynthesis in kilonovae (Drout et al. 2017; Smartt et al. 2017), new insights into gamma-ray burst mechanisms (Mooley et al. 2018; Nakar et al. 2018), constraints on the neutron star equation of state (Abbott et al. 2018b; Raithel et al. 2018), and even an independent measurement of the Hubble constant (Abbott et al. 2017c; Hotokezaka et al. 2018).

Beyond the electromagnetic counterparts associated with GW170817, binary neutron star mergers are predicted to be accompanied by prompt radio emission (Usov \& Katz 2000; Hansen \& Lyutikov 2001; Pshirkov \& Postnov 2010; Lai 2012; Lyutikov 2013, 2019; Totani 2013; Ravi \& Lasky 2014; Metzger \& Zivancev 2016; Wang et al. 2016, 2018). Unlike the late-time radio afterglow associated with GW170817, due to the interaction of relativistic ejecta with the ambient medium, the theorized prompt radio emission is generated by processes internal to the merging objects themselves. In particular, prompt emission may take the form of a short (likely subsecond) coherent radio pulse generated near the instant of merger.

The detection of prompt radio emission from a binary neutron star would yield an immense amount of information, probing the binary's immediate magnetic environment near the time of merger, tracing properties of the intergalactic medium, and offering rapid arcminute constraints on the progenitor's location. However, the observation of prompt emission is made difficult by several factors (Yancey et al. 2015; Chu et al. 2016; Kaplan et al. 2016). First, gravitational-wave detectors provide only poor localization of gravitational-wave sources. Even for the three-detector Advanced Laser Interferometer Gravitational-Wave Observatory (LIGO) and Virgo network, the median binary neutron star localization is expected to be 120-180 deg ${ }^{2}$ during the upcoming O3 observing run (Abbott et al. 2018a). Second, low-frequency prompt emission released at time of merger may arrive at Earth as little as one minute after the gravitational-wave signal, slowed only by free electrons encountered during propagation. Searches for prompt radio emission are therefore typically limited by the latency with which gravitational-wave candidates are announcednotices released more than minutes after a gravitational wave's arrival may well come too late.

All previous searches for prompt radio emission have targeted short gamma-ray bursts (Bannister et al. 2012; Obenberger et al. 2014; Kaplan et al. 2015; Anderson et al. 2018; Rowlinson \& Anderson 2019) or were carried out too late to detect any prompt emission that may have been present (Callister et al. 2017; Kaplan et al. 2017). Here, we perform the first search for prompt radio emission coincident with a gravitational-wave signal, using the Owens Valley Radio Observatory Long Wavelength Array (OVRO-LWA). Observing between 27 and $84 \mathrm{MHz}$, the OVRO-LWA consists of 288 dual-polarization antennas spanning $\sim 1.5 \mathrm{~km}$. Cross correlation of 256 antennas with the LargeAperture Experiment to Detect the Dark Age correlator provides 
all-sky imaging with $24 \mathrm{kHz}$ frequency resolution and $\sim 10^{\prime}$ spatial resolution (Kocz et al. 2015; Anderson et al. 2018; Eastwood et al. 2018).

The OVRO-LWA is uniquely suited to the challenge of detecting prompt radio emission. Its nearly hemispherical field of view can capture much of the LIGO-Virgo localization region within a single image. Additionally, the OVRO-LWA operates in a continuous buffered mode, temporarily saving all visibilities to disk for up to $24 \mathrm{hr}$. This alleviates (although does not eliminate; see Section 5) the need for rapid LIGO-Virgo notices. Provided that a notice is released within one day of the gravitational-wave event, the relevant on-source data can be retrieved from the buffer and written to disk.

Although the OVRO-LWA was observing at the time of GW170817, the binary neutron star merger occurred below the OVRO-LWA's horizon (Abbott et al. 2017a). We therefore cannot place any observational limits on the prompt radio emission associated with GW170817. Instead, we report the results of a search for prompt radio emission associated with the binary black hole merger GW170104 (Abbott et al. 2017d).

Although stellar-mass binary black hole mergers are generally not expected to yield electromagnetic transients, the tentative Fermi-GBM detection of gamma-rays associated with the binary black hole merger GW150914 (Abbott et al. 2016; Connaughton et al. 2016) has sparked new interest in possible counterparts to LIGO/Virgo's binary black hole events. In particular, binary black holes might conceivably generate electromagnetic transients if one or more of the black holes is charged (Liebling \& Palenzuela 2016; Liu et al. 2016; Zhang 2016; Fraschetti 2018), in the presence of a circumstellar or circumbinary disk (Perna et al. 2016; de Mink \& King 2017), or in the case of black hole "twins" born from the collapse of a single massive star (Loeb 2016). Although the statistical significance of the Fermi-GBM candidate remains under debate (Greiner et al. 2016; Lyutikov 2016; Savchenko et al. 2016; Connaughton et al. 2018), the plethora of models predicting electromagnetic counterparts makes binary black hole mergers an interesting (if speculative) observational target.

While valuable in its own right, the search for prompt radio emission from GW170104 additionally serves as a powerful proof of principle. GW170104 exemplifies the challenges facing detection of prompt radio emission. First, its accompanying localization is poor, spanning a significant fraction of the sky. Second, the LIGO/Virgo alert announcing the detection of GW170104 was released hours after the gravitational-wave event, long after the expected arrival of any prompt radio emission. Despite these challenges, we place stringent upper limits on the prompt radio luminosity of GW170104, demonstrating the capability of the OVRO-LWA to follow up future compact binary mergers.

In Section 2 below, we begin by describing the gravitationalwave signal GW170104 as well as the OVRO-LWA data collection and initial reduction. In Section 3, we then describe the search for a dispersed radio transient, and in Section 4 present upper limits on the radio flux and luminosity of GW170104. Finally, in Section 5 we discuss future prospects for the follow-up of gravitational-wave candidates.

\section{GW170104 and OVRO-LWA Observations}

The gravitational-wave signal GW170104 was measured on 2017 January 4 at 10:11:58.6 UTC by the Advanced LIGO

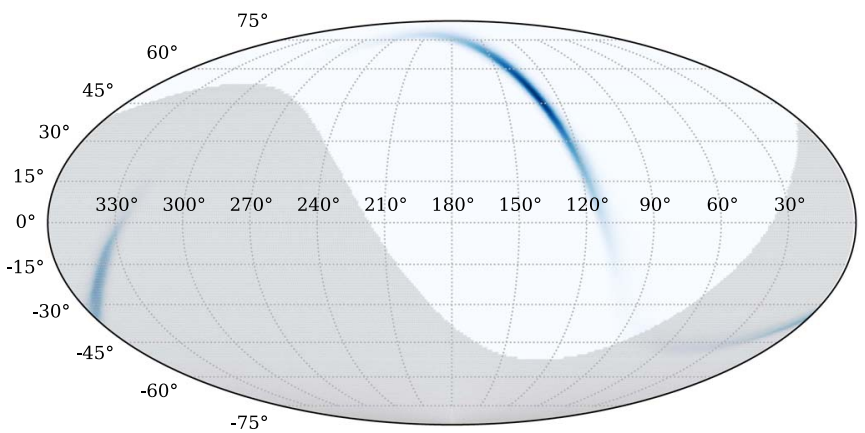

Figure 1. Posterior probability distribution (in blue) on the sky position of the binary black hole merger GW170104. Also shown is the OVRO-LWA's field of view at GW170104's time of arrival; areas below the OVRO-LWA horizon are shaded in gray.

experiment (Abbott et al. 2017d). Arising from a $31+19 M_{\odot}$ binary black hole merger, the signal was initially localized to a $\sim 1600$ square degree band on the sky (see Figure 1) and its redshift estimated to be $z=0.18_{-0.07}^{+0.08}$. Following a delay related to the calibration of Advanced LIGO's Hanford detector, an alert with preliminary event localization was released at 16:49:56 UTC, six hours after the gravitational wave's arrival (Abbott et al. 2019).

At this time, the OVRO-LWA was under continuous operation, temporarily storing $13 \mathrm{~s}$ integrations in a continuously overwritten $24 \mathrm{hr}$ buffer. Upon receiving the gravitational-wave event notice, buffered data spanning 09:00:03 to 14:11:11 UTC were copied to long-term storage.

Data are flagged on a per antenna, baseline, and channel basis. We flag antennas showing anomalous autopower spectra, cutting an average of 54 antennas ( $~ 38 \%$ of visibilities). An additional 398 baselines are flagged to mitigate cross-talk between adjacent signal paths and eliminate other spurious excess power. Finally, loud individual channels are automatically flagged to reduce radio frequency interference (RFI) removing $\sim 12 \%$ of the 2398 frequency channels.

Cassiopeia (Cas) A and Cygnus (Cyg) A are the brightest sources in the low-frequency radio sky and therefore make opportune calibration sources. We calibrate our visibility data using a single integration recorded roughly 10 hours earlier, at 22:44:04 January 3 UTC (21:49:47 local sidereal time), when both Cas A and Cyg A are close to zenith. The per-channel complex gains of each antenna are determined using a simplified sky model comprising three point sources-Cas A, Cyg A, and the Sun (Baars et al. 1977; Perley \& Butler 2017).

Following this initial calibration, there persist residual errors due to unmodeled directional variations in antenna gains. To combat sidelobe contamination arising from these errors, we "peel" bright sources, performing an additional direction-dependent calibration and subtraction of these sources (Eastwood 2016). At the time of GW170104, Cas A and Taurus (Tau) A are the brightest sources in the OVRO-LWA field of view (Cyg A had since set below the horizon). We peel both Cas A and Tau A, as well as a generic near-field source to remove a stationary noise pattern likely caused by cross-talk between electronics (Eastwood et al. 2018). Because Cas A is nearly on the OVRO-LWA's horizon, this peeling procedure fails for a small number of integrations; these integrations are manually flagged.

Figure 2 shows a peeled and deconvolved $13 \mathrm{~s}$ image of the OVRO-LWA sky at the time of GW170104 with 0.125 


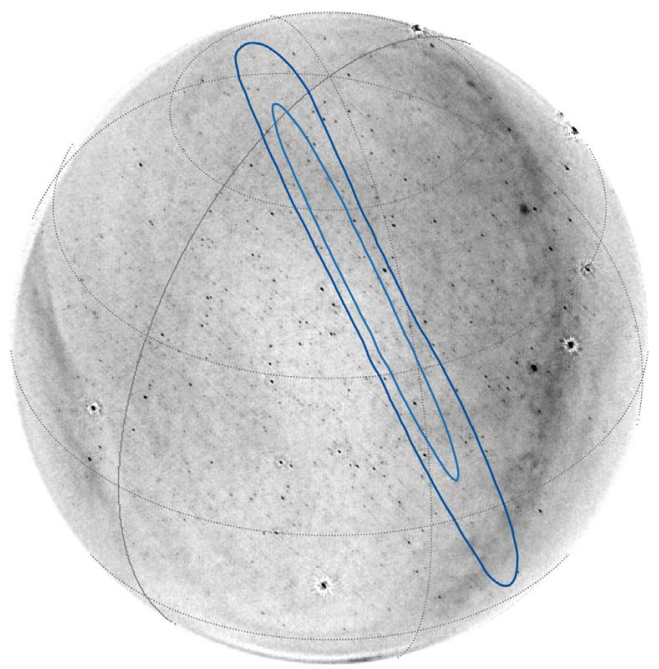

Figure 2. Total intensity image of the OVRO sky from 27 to $84 \mathrm{MHz}$ in a $13 \mathrm{~s}$ interval centered at 10:11:54.1 UTC, containing GW170104's time of arrival. The dark and light blue contours show the $95 \%$ and $68 \%$ credible bounds on the location of GW170104's progenitor, respectively, conditioned on the OVRO-LWA's field of view.

resolution. Deconvolution is performed using the wsclean algorithm with a Briggs weighting of 0 and a multiscale bias of 0.6 (Offringa et al. 2014). The blue contours show the $68 \%$ and $95 \%$ credible bounds on the sky location of GW170104's progenitor, restricted to the OVRO-LWA's field of view. The $95 \%$ credible contour contains 72,556 pixels, each of which we search for a dispersed radio signal.

\section{Search for a Dispersed Signal}

Radio waves of frequency $\nu$ propagating through the interstellar and/or intergalactic media experience a dispersion delay

$$
\begin{aligned}
t & =\left(\frac{e^{2}}{2 \pi m_{e} c}\right) \frac{\mathrm{DM}}{\nu^{2}} \\
& =\left(4.149 \times 10^{3} \mathrm{~s}\right)\left(\frac{\mathrm{DM}}{\mathrm{pc} \mathrm{cm}^{-3}}\right)\left(\frac{\nu}{\mathrm{MHz}}\right)^{-2}
\end{aligned}
$$

relative to signals of infinite frequency. Here, $e$ is the fundamental charge, $m_{e}$ is the electron mass, $c$ is the speed of light, and the dispersion measure DM is the integrated column density of free electrons along the wave's path. The dispersion measure may contain contributions from the immediate environment and/or host galaxy of GW170104, the intergalactic medium, and the interstellar medium of the Milky Way. Using gravitational-wave constraints on GW170104's redshift and sky location (and somewhat arbitrarily budgeting up to $100 \mathrm{pc} \mathrm{cm}^{-3}$ for GW170104's unknown environment and host), we bound the dispersion measure of an associated radio transient to $113 \mathrm{pc} \mathrm{cm}^{-3} \leqslant \mathrm{DM} \leqslant 630 \mathrm{pc} \mathrm{cm}^{-3}$, corresponding to time delays ranging from 640 to $3600 \mathrm{~s}$ at the bottom of the OVRO-LWA band (see Appendix A for details). We therefore analyze data up to one hour after the gravitational-wave event. Some models for prompt radio emission predict a precursor signal released before binary merger (Hansen \& Lyutikov 2001; Lyutikov 2013, 2019; Metzger \& Zivancev 2016;
Wang et al. 2018), and so we additionally analyze the 70 minutes of buffered data recorded before the event. Our final data set comprises 610 integrations spanning 09:00:03 to 11:12:00 UTC, each $13 \mathrm{~s}$ in duration.

Note that astrophysical signals will also be dispersed within each frequency channel. This intra-channel dispersion is strongest in the lowest channel, in which signals separated by the $24 \mathrm{kHz}$ bandwidth are delayed by a maximum of $6.4 \mathrm{~s}$ with respect to one another. Although this delay is smaller than our $13 \mathrm{~s}$ integration time, it is sufficiently long that a randomly placed transient might conceivably be split across adjacent integrations, potentially degrading our search sensitivity at low frequencies.

Scatter broadening is unlikely to affect our search. Assuming a $\nu^{-4}$ frequency dependence, the estimated Milky Way scattering timescale of $0.06 \mu \mathrm{s}$ at $1 \mathrm{GHz}$ corresponds only to $0.1 \mathrm{~s}$ at $28 \mathrm{MHz}$, much less than our $13 \mathrm{~s}$ integration time (Cordes \& Lazio 2002). We might expect similarly negligible contributions from GW170104's host galaxy. Additionally, fast radio bursts show minimal scattering due to the intergalactic medium (Cordes et al. 2016).

Our search window spans approximately 130 minutes. In this time the sky rotates considerably, and so we must track the movement of a given source across the OVRO-LWA's field of view. Just as a sufficiently broadened pulse could span multiple time integrations at a given frequency, it is possible for the Earth's rotation to smear emission across multiple image pixels within a single $13 \mathrm{~s}$ integration. Because the array's synthesized beam (with 0.50 and 0.24 major and minor axes at $56 \mathrm{MHz}$ ) is larger than our $0^{\circ} .125$ pixel size, any astrophysical emission will manifest in multiple neighboring pixels. We are therefore unlikely to miss a significant fraction of a source's emission as we follow it from one image pixel to the next.

As an example, Figure 3 shows the dynamic spectrum obtained by tracking a randomly chosen location within the GW170104 localization region. To account for slow temporal variations and sidelobes from bright, nearby sources, we have subtracted away the median flux measured in an annulus extending five to seven beamwidths around the target location (see Figure 6 below). We search all such dynamic spectra for significant dispersed transients, stepping through dispersion measures and times $t_{0}$ at which a proposed signal enters the OVRO-LWA band. The spacing $\delta \mathrm{DM}$ between our dispersion measure trials is set by our $t_{\text {int }}=13 \mathrm{~s}$ integration time and the bounds $\nu_{1}=27.384 \mathrm{MHz}$ and $\nu_{2}=84.912 \mathrm{MHz}$ on the OVRO-LWA band:

$$
\frac{\delta \mathrm{DM}}{\mathrm{pc} \mathrm{cm} \mathrm{cm}^{-3}}=\frac{t_{\mathrm{int}}}{4.149 \times 10^{3} \mathrm{~s}}\left[\left(\frac{\nu_{1}}{\mathrm{MHz}}\right)^{-2}-\left(\frac{\nu_{2}}{\mathrm{MHz}}\right)^{-2}\right]^{-1},
$$

giving $\delta \mathrm{DM}=2.62 \mathrm{pc} \mathrm{cm}^{-3}$. For each dispersed track, we estimate the corresponding flux density with the weighted average

$$
\hat{F}=\frac{\sum_{i} \hat{F}_{i} / \sigma_{i}^{2}}{\sum_{j} 1 / \sigma_{j}^{2}}
$$

where $\hat{F}_{i}$ is the measured flux density in the track's $i$ th timefrequency pixel and $\sigma_{i}^{2}$ is the corresponding variance, estimated using the background annulus. In the presence of a true radio transient of flux density $F$, the expectation value and variance 


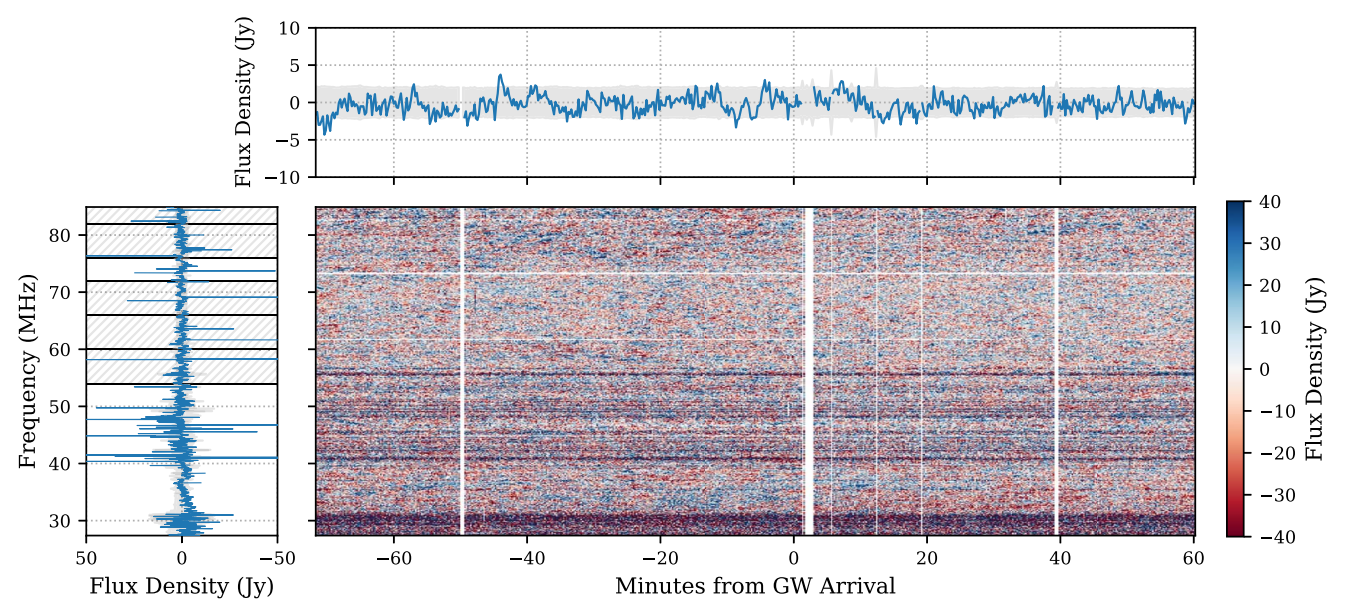

Figure 3. Dynamic spectrum of a randomly chosen sky location within the GW170104 localization region (Figure 2), after subtraction of the median flux measured in an annulus surrounding the target location. White vertical and horizontal bands correspond to times and frequency channels that have been flagged due to excess antenna power or RFI. The left and upper subplots show the time- and frequency-averaged flux densities, respectively. The filled gray region within each subplot marks the $\pm 3 \sigma$ band as measured in the background annulus. Broadcast television channels are denoted by hatched regions in the time-averaged spectrum; these channels represent common sources of RFI due to meteor reflection events.

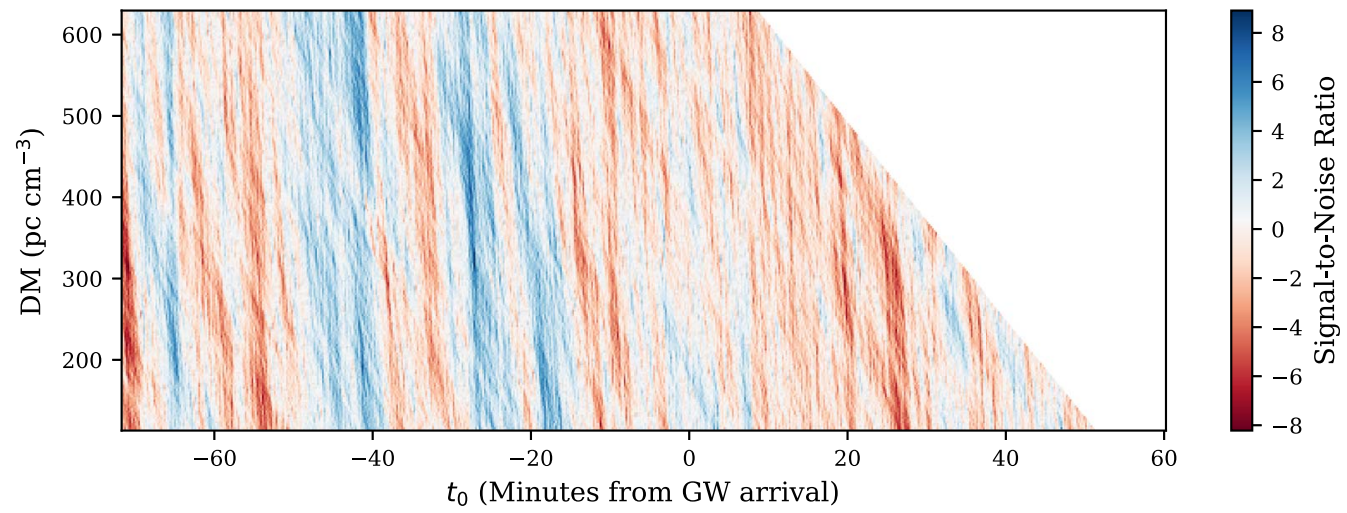

Figure 4. Signal-to-noise ratios as a function of dispersion measure DM and the initial time $t_{0}$ at which a signal is presumed to enter the OVRO-LWA band, targeting the same sky location as Figure 3. As described in Section 3, we search for signals with dispersion measures $113 \mathrm{pc} \mathrm{cm}^{-3} \leqslant \mathrm{DM} \leqslant 630 \mathrm{pc} \mathrm{cm}^{-3}$ in a roughly onehour window around the GW170104's time of arrival. The blank region on the figure's right-hand side corresponds to time-frequency tracks that extend beyond the duration of our data set.

of $\hat{F}$ are

$$
\langle\hat{F}\rangle=F
$$

and

$$
\sigma^{2}=\frac{1}{\sum_{i} 1 / \sigma_{i}^{2}},
$$

respectively. The signal-to-noise ratio $(\mathrm{S} / \mathrm{N})$ of each dispersion trial is defined by combining Equations (3) and (5):

$$
\mathrm{S} / \mathrm{N}=\frac{\hat{F}}{\sigma}
$$

Figure 4, for example, shows the $\mathrm{S} / \mathrm{Ns}$ obtained from dedispersing the dynamic spectrum in Figure 3.

With 72,556 sky pixels and 92,763 DM and $t_{0}$ trials per pixel, a dedispersion search over the entire GW170104 localization region yields $6.7 \times 10^{9}$ total trials. To determine a suitable $\mathrm{S} / \mathrm{N}$ threshold for manual follow-up, in Figure 5 we plot the cumulative distribution of $\mathrm{S} / \mathrm{Ns}$ obtained from a

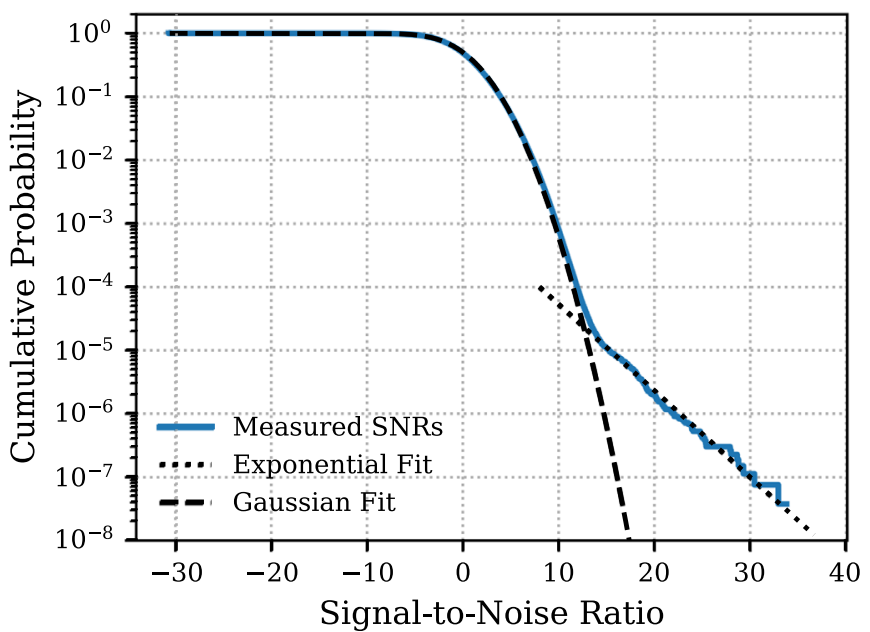

Figure 5. Cumulative background distribution of $\mathrm{S} / \mathrm{Ns}$ from a subset of sky directions and dispersion trials. The distribution is well fit by a central Gaussian and a exponential tail dominated by meteor reflection events. Based on this distribution, we manually follow up any dispersion trial giving $\mathrm{S} / \mathrm{N}>20$. 

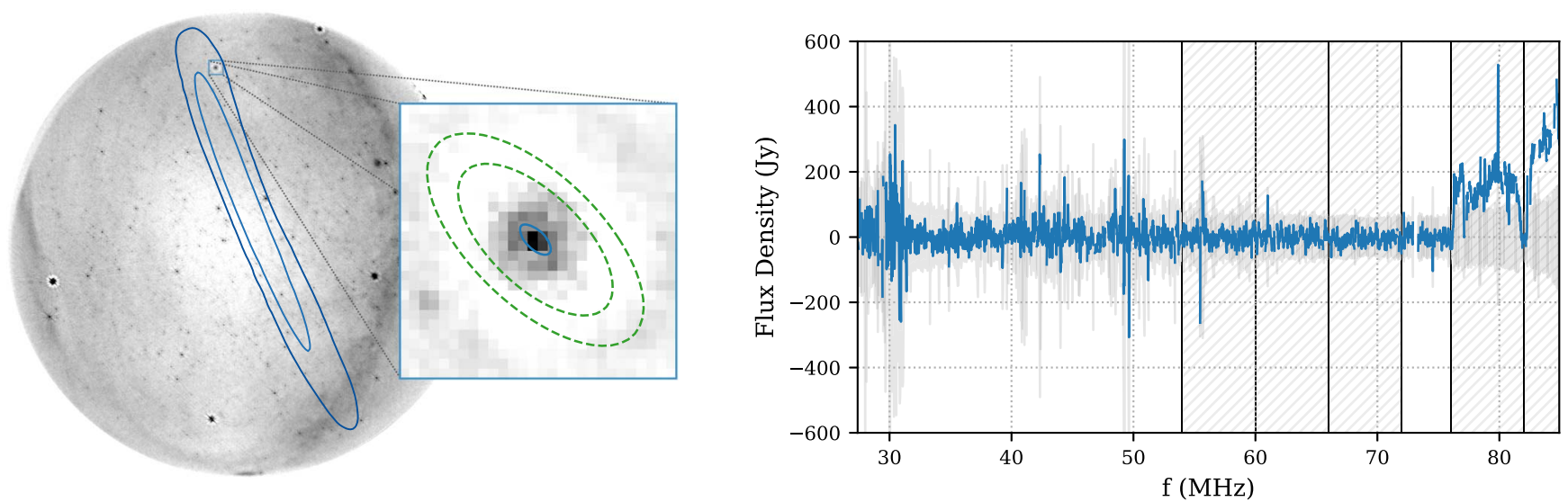

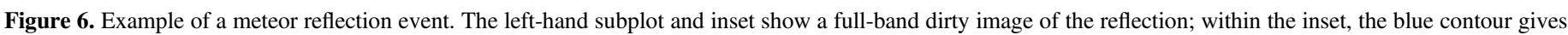

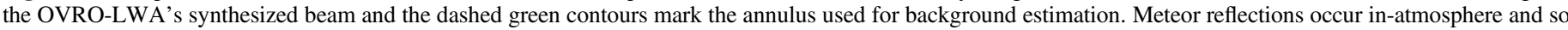

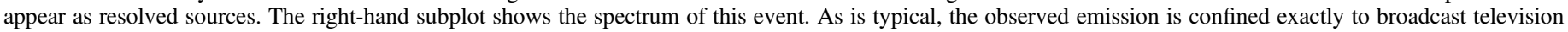
channels $5(76-82 \mathrm{MHz})$ and $6(82-88 \mathrm{MHz})$, and so is readily identifiable as terrestrial in origin.

random subset of sky locations, dispersion measures, and initial times $t_{0}$. We find our $\mathrm{S} / \mathrm{Ns}$ to be fairly Gaussian distributed. The bulk of the distribution is well fit by a somewhat broadened Gaussian centered at zero with a variance of 1.44. At high significances, however, Figure 5 shows the emergence of a non-Gaussian tail. This tail is dominated by meteor reflection events, in which patches of atmosphere temporarily ionized by passing meteors act as reflective surfaces, redirecting RFI from beyond the horizon into the OVRO-LWA (see more below). The tail is well fit by $\log _{10} P=a(\mathrm{~S} / \mathrm{N})+b$, with $a=-0.136$ and $b=-2.913$. Using this fit, we choose our threshold for manual inspection to be $\mathrm{S} / \mathrm{N}=20$, above which we expect $\left(6.7 \times 10^{9}\right) 10^{a(\mathrm{~S} / \mathrm{N})+b} \approx 1.5 \times 10^{4}$ outliers.

After searching across the entire GW170104 localization region, we find 6828 outliers exceeding our threshold. This suggests that extrapolation of the subset of data shown in Figure 5 overestimates the rate of high significance events by a factor of two. All candidates warranting manual follow-up are identified as meteor reflection events (Ceplecha et al. 1998; Helmboldt et al. 2014). Figure 6 illustrates the properties of a typical reflection event. First, meteor reflections occur within the atmosphere (well inside the array's $2 D^{2} / \lambda \sim 1000 \mathrm{~km}$ farfield limit) and hence appear as resolved sources. Second, their spectra show emission confined to one or more broadcast television channels. The reflection event in Figure 6 , for instance, is confined to channels $5(76-82 \mathrm{MHz})$ and 6 $(82-88 \mathrm{MHz})$. As meteor reflections currently dominate our search background, the automated identification and rejection of meteor reflections will be a crucial step in improving the sensitivity of future searches.

\section{Radio Luminosity Limits}

Having rejected all outliers as reflection events, we place upper limits on the prompt radio emission associated with GW170104, following the Bayesian approach described in Appendix B. Figure 7 shows our $95 \%$ credible flux upper limits for each pixel within the $95 \%$ credible gravitational-wave localization region. We exclude pixels containing persistent point sources detected at $5 \sigma$ prior to dedispersion, yielding the "holes" seen in Figure 7. We additionally trim the southernmost points that set below the OVRO-LWA's horizon during the observation. All together, we cover $94 \%$ of the localization region contained within the OVRO-LWA's field of view, and

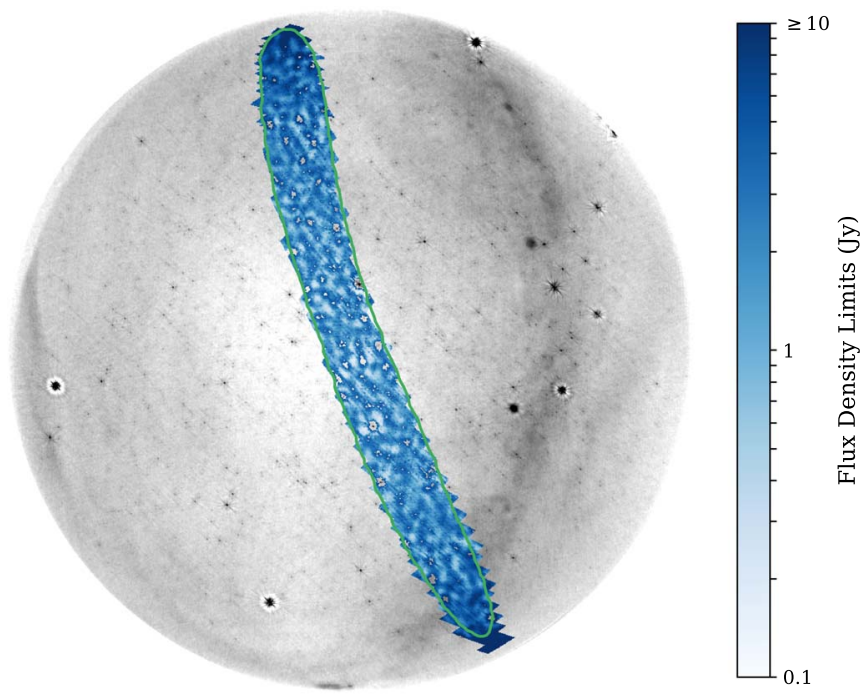

Figure 7. $95 \%$ credible upper limits on the flux density of prompt radio emission from GW170104, as a function of its presumed sky location. The "holes" mark locations of persistent point sources excluded from our analysis. For reference, the contour traces the 95\% credible localization of GW170104 within the OVRO-LWA's field of view. Our median upper limit across the sky is $2.4 \mathrm{Jy}$. Marginalizing over the sky location and distance constraints due to the gravitational-wave signal, we limit GW170104's equivalent isotropic luminosity between 27 and $84 \mathrm{MHz}$ to $L \leqslant 2.5 \times 10^{41} \mathrm{erg} \mathrm{s}^{-1}$ at $95 \%$ credibility.

$54 \%$ of GW170104's global probability map. We achieve a median upper limit of $2.4 \mathrm{Jy}$. Our sensitivity is degraded at low elevations due to the $(\sin \theta)^{1.6}$ scaling of the antennas' primary beam with elevation angle $\theta$ (Hicks et al. 2012). Flux upper limits are also impacted by sidelobes in the vicinity of particularly bright point sources.

With the sky and distance localization provided by Advanced LIGO, we can re-express our flux limits as constraints on the equivalent isotropic radio luminosity of GW170104. Marginalizing over the sky location and distance of GW170104 (see Appendix B), we limit its equivalent isotropic luminosity between 27 and $84 \mathrm{MHz}$ to $L_{\text {radio }} \leqslant 2.5 \times 10^{41} \mathrm{erg} \mathrm{s}^{-1}$ at $95 \%$ credibility, assuming the source lies within the OVRO-LWA's field of view. The total energy radiated by GW170104 was $E_{\mathrm{GW}}=2.0_{-0.7}^{+0.6} M_{\odot} c^{2}$ (Abbott et al. 2017d). We therefore limit 
the fraction of the total energy converted to prompt radio emission to $L_{\text {radio }} t_{\text {int }} / E_{\mathrm{GW}} \leqslant 1.4 \times 10^{-12}$, using the lower bound on $E_{\mathrm{GW}}$.

For reference, the equivalent isotropic luminosity of the Fermi-GBM outlier associated with GW150914 was $1.8_{-1.0}^{+1.5} \times$ $10^{49} \mathrm{erg} \mathrm{s}^{-1}$ (Connaughton et al. 2016). Note that GW150914 and GW170104 occurred at luminosity distances of approximately $410 \mathrm{Mpc}$ and $880 \mathrm{Mpc}$, respectively (Abbott et al. 2016, 2017d). If the OVRO-LWA had been operating at the time of GW150914, we would therefore have been sensitive to any associated radio transient with luminosity $2.5 \times 10^{41} \mathrm{erg} \mathrm{s}^{-1}$ $(410 \mathrm{Mpc} / 880 \mathrm{Mpc})^{2} \approx 5.5 \times 10^{40} \mathrm{erg} \mathrm{s}^{-1}$. Hence in the future, if additional gamma-ray outliers are identified in coincidence with gravitational-wave events, simultaneous observations with the OVRO-LWA will limit the ratio of radio and gamma-ray luminosities to $\lesssim 3 \times 10^{-9}$.

Similar limits will be possible for future binary neutron star mergers. GW170817 occurred at a distance of $40.7 \mathrm{Mpc}$ (Cantiello et al. 2018). OVRO-LWA follow-up of binary neutron stars at comparable distances will yield luminosity limits of $2.5 \times 10^{41} \mathrm{erg} \mathrm{s}^{-1}(40.7 \mathrm{Mpc} / 880 \mathrm{Mpc})^{2} \approx 5 \times$ $10^{38} \mathrm{erg} \mathrm{s}^{-1}$. Meanwhile, the equivalent isotropic luminosity of GRB 170817A was estimated to be $1.6 \times 10^{47} \mathrm{erg} \mathrm{s}^{-1}$ (with total energy $3.1 \times 10^{46} \mathrm{erg}$ ) in the $1 \mathrm{keV}-10 \mathrm{MeV}$ band (Abbott et al. 2017e). The limits attainable with the OVROLWA would therefore limit the ratio of radio and gamma-ray luminosities to $\lesssim 3 \times 10^{-9}$, and the ratio of total radiated energies to $\lesssim 2 \times 10^{-7}$.

\section{The Third LIGO/Virgo Observing Run and Beyond}

Advanced LIGO and Virgo's third observing run (O3) began in 2019 April and is scheduled to run for one calendar year. During this time, between one and 50 binary neutron star detections are expected (Abbott et al. 2018a). The OVROLWA will operate in continuous buffering mode during O3, searching for prompt radio transients associated with compact binary mergers.

The sensitivity of this study to sub-second radio transients is limited by the 13 s resolution of buffered visibilities Rowlinson \& Anderson (2019). The buffering of future data with higher time resolution will increase the signal-to-noise ratio of temporally unresolved transients. We are additionally exploring options to buffer the incoherent sum of antenna powers at their raw $197 \mathrm{MHz}$ sampling rate. The incoherent sum will provide no directional information, but the vastly increased time resolution and temporal coincidence with gravitational-wave events will enable sensitive measurements of prompt radio transients.

A more ambitious goal is the buffering and coherent dedispersion of all 512 signal paths at $197 \mathrm{MHz}$. This endeavor has previously required prohibitively large buffer disk space due to significant latency in the release of LIGO/Virgo alerts. In their upcoming $\mathrm{O} 3$ observing run, however, LIGO and Virgo will transition to automated alerts released within 1-10 minutes of a gravitational-wave candidate (LIGO Scientific Collaboration \& Virgo Collaboration 2018). If successful, this reduced latency may make the buffering of raw antenna voltages computationally feasible.

Finally, the OVRO-LWA will soon be undergoing upgrades toward its "Stage 3" design, consisting of 352 correlated antennas over an extended $2.5 \mathrm{~km}$ maximum baseline. Also included in this design is the buffering of raw antenna voltages, allowing high time-resolution searches triggered by automated LIGO and Virgo alerts. With these improvements, Stage 3 OVRO-LWA promises to enable even more sensitive detection and precise localization of prompt radio emission from compact binary mergers.

This material is based in part upon work supported by the National Science Foundation under grant AST-1654815 and AST-1212226. T.C. is supported by LIGO Laboratory, funded by the National Science Foundation under cooperative agreement PHY-0757058, and by the Josephine de Karman Fellowship Trust. G.H. acknowledges the support of the Alfred P. Sloan Foundation and the Research Corporation for Science Advancement. Part of this research was carried out at the Jet Propulsion Laboratory, California Institute of Technology, under a contract with the National Aeronautics and Space Administration. The OVRO-LWA project was initiated through the kind donation of Deborah Castleman and Harold Rosen.

\section{Appendix A Dispersion Measure Bounds}

Parameter estimation on the gravitational-wave signal GW170104 constrains its redshift to $z=0.173_{-0.071}^{+0.072}$ with an effective-precession waveform model and $z=0.182_{-0.078}^{+0.081}$ using a model capturing full spin-precession effects (Abbott et al. 2017d). We conservatively assume that GW170104's progenitor lies between $0.1 \leqslant z \leqslant 0.3$.

Dispersion measure is defined as the integrated column density of free electrons (number density $n_{e}$ ) along a given line of sight: $\mathrm{DM}=\int n_{e} d s$. When allowing for cosmology, the dispersion measure due to propagation through the intergalactic medium is (Ioka 2003; Inoue 2004)

$$
\mathrm{DM}_{\mathrm{IGM}}=\bar{n}_{e} c \int_{0}^{z} \frac{\left(1+z^{\prime}\right)}{H\left(z^{\prime}\right)} d z^{\prime},
$$

where $H(z)=H_{0} \sqrt{\Omega_{m}(1+z)^{3}+\Omega_{\Lambda}}, \quad H_{0}$ is the Hubble constant, and $\Omega_{m}$ and $\Omega_{\Lambda}$ are the dimensionless energy-densities of matter and dark energy, respectively. We take $H_{0}=$ $67.7 \mathrm{~km} \mathrm{~s}^{-1} \mathrm{Mpc}^{-1}, \Omega_{m}=0.31$, and $\Omega_{\Lambda}=0.69$. An upper limit on mean electron density in the intergalactic medium is obtained by assuming the universe's baryonic density $\Omega_{B}=0.049$ is composed entirely of ionized hydrogen (Ioka 2003). Then the mean electron number density is $\bar{n}_{e}=\Omega_{B} \rho_{c} / m_{p}$, where $\rho_{c}=$ $3 H_{0}^{2} / 8 \pi G$ is the closure density of the universe, $G$ is Newton's constant, and $m_{p}$ is the proton mass. As the universe is neither fully ionized nor composed purely of hydrogen, this approximation yields an overestimate of $\bar{n}_{e}$ and hence a conservative overestimate of the intergalactic dispersion measure. Assuming that GW170104's progenitor lies within $0.1 \leqslant z \leqslant 0.3$, we estimate $113 \mathrm{pc} \mathrm{cm}^{-3} \leqslant$ DMIGM $\leqslant 350 \mathrm{pc} \mathrm{cm}^{-3}$.

In addition, the Milky Way, the progenitor's host galaxy, and the progenitor's immediate environment will contribute to the net dispersion measure. The GW170104 localization region spans a broad range of Galactic latitudes, corresponding to a wide range of possible Galactic dispersion measures. A lower bound on the Milky Way dispersion measure is simply zero. An upper bound is given by assuming a line of sight directly through the Galactic disk, yielding $0 \leqslant \mathrm{DM}_{\mathrm{MW}} \leqslant 180 \mathrm{pc} \mathrm{cm}^{-3}$ (Cordes \& Lazio 2002, 2003). We have no knowledge of the progenitor's host galaxy or its environment, and so we naively 
assume $\mathrm{DM}_{\mathrm{Host}}+\mathrm{DM}_{\mathrm{Env}} \leqslant 100 \mathrm{pc} \mathrm{cm}^{-3}$. Compact binaries are expected to be spatially offset from their host galaxy due to natal supernova kicks or dynamical ejection from dense clusters (Berger 2014; Rodriguez et al. 2018), and so in practice the dispersion measure from the binary's immediate environment may dominate over that from its host.

Combining contributions from the Milky Way, the intergalactic medium, and GW170104's environment and host galaxy, we bound the dispersion measure of radio transients associated with $\mathrm{GW} 170104$ to $113 \mathrm{pc} \mathrm{cm}^{-3} \leqslant \mathrm{DM}_{\mathrm{IGM}} \leqslant$ $630 \mathrm{pc} \mathrm{cm}^{-3}$.

\section{Appendix B}

\section{Flux and Luminosity Upper Limits}

Given radio data, which we will represent via $\boldsymbol{d}$, our first goal is to compute the posterior $p(F \mid d, \hat{\Omega})$ on the radio flux of GW170104 for every possible sky location. This posterior is obtained by marginalizing over dispersion measure and initial signal time:

$$
p(F \mid \boldsymbol{d}, \hat{\Omega})=\int d \mathrm{DM} \int d t_{0} p\left(F \mid \boldsymbol{d}, \hat{\Omega}, \mathrm{DM}, t_{0}\right) p(\mathrm{DM}) p\left(t_{0}\right),
$$

where $p(\mathrm{DM})$ and $p\left(t_{0}\right)$ are our prior probabilities on a signal's dispersion measure and initial time. Next, using Bayes' theorem, we can relate the posterior $p\left(F \mid \boldsymbol{d}, \hat{\Omega}, \mathrm{DM}, t_{0}\right)$ to the likelihood $p\left(\boldsymbol{d} \mid F, \hat{\Omega}, \mathrm{DM}, t_{0}\right)$ of having measured $\boldsymbol{d}$ :

$$
\begin{aligned}
p(F \mid \boldsymbol{d}, \hat{\Omega}) \propto & \int d \mathrm{DM} \int d t_{0} p\left(\boldsymbol{d} \mid F, \hat{\Omega}, \mathrm{DM}, t_{0}\right) \\
& \times p(F) p(\mathrm{DM}) p\left(t_{0}\right),
\end{aligned}
$$

where $p(F)$ is our flux density prior.

We assume Gaussian likelihoods, centered at $\hat{F}\left(\hat{\Omega}, \mathrm{DM}, t_{0}\right)$ and with variance $\sigma^{2}\left(\hat{\Omega}, \mathrm{DM}, t_{0}\right)$; see Equations (3) and (5). Meanwhile, for simplicity we assume flat priors over the ranges $\Delta \mathrm{DM}$ and $\Delta t_{0}$ considered: $p(\mathrm{DM})=1 / \Delta \mathrm{DM}$ and $p\left(t_{0}\right)=1 / \Delta t_{0}$. We similarly assume a uniform (improper) prior over all positive $F$. All together,

$$
\begin{aligned}
p(F \mid \boldsymbol{d}, \hat{\Omega})= & \int \frac{d \mathrm{DM}}{\Delta \mathrm{DM}} \int \frac{d t_{0}}{\Delta t_{0}} \frac{1}{\mathcal{N}\left(\hat{\Omega}, \mathrm{DM}, t_{0}\right)} \\
& \times \exp \left(-\frac{\left[\hat{F}\left(\hat{\Omega}, \mathrm{DM}, t_{0}\right)-F\right]^{2}}{2 \sigma^{2}\left(\hat{\Omega}, \mathrm{DM}, t_{0}\right)}\right),
\end{aligned}
$$

with normalization factor

$$
\mathcal{N}\left(\hat{\Omega}, \mathrm{DM}, t_{0}\right)=\int_{0}^{\infty} d F \exp \left(-\frac{\left[\hat{F}\left(\hat{\Omega}, \mathrm{DM}, t_{0}\right)-F\right]^{2}}{2 \sigma^{2}\left(\hat{\Omega}, \mathrm{DM}, t_{0}\right)}\right) .
$$

With the flux posterior $p(F \mid \boldsymbol{d}, \hat{\Omega})$ in hand, the $95 \%$ credible flux upper limit in direction $\hat{\Omega}$ corresponds to the flux $F_{95}$ satisfying

$$
0.95=\int_{0}^{F_{95}} d F p(F \mid d, \hat{\Omega}) .
$$

These upper limits are shown in Figure 7 above.

Using the Advanced LIGO posterior on GW170104's location, we can additionally compute a posterior $p(L \mid \boldsymbol{d})$ on the equivalent isotropic luminosity of GW170104, marginalized over all possible progenitor sky locations $\hat{\Omega}$ and distances $D$ :

$$
p(L \mid \boldsymbol{d})=\int d D \int d \hat{\Omega} p(L \mid \boldsymbol{d}, D, \hat{\Omega}) p(D, \hat{\Omega}) .
$$

Here, $p(D, \hat{\Omega})$ is the probability distribution on the progenitor location of GW170104; we take this to be the localization provided by Advanced LIGO. As in Equation (12) above, the $95 \%$ credible upper limit is given by the luminosity $L_{95}$ satisfying $0.95=\int_{0}^{L_{95}} p(L \mid \boldsymbol{d}) d L$, or

$$
0.95=\int d D \int d \hat{\Omega} \int_{0}^{L_{95}} d L p(L \mid d, D, \hat{\Omega}) p(D, \hat{\Omega}) .
$$

Note that, as currently written, this equation requires posterior probabilities $p(L \mid \boldsymbol{d}, D, \hat{\Omega})$ on luminosity as a function of direction and distance. We can recast Equation (14) in terms of our known flux posteriors $p(F \mid \boldsymbol{d}, \hat{\Omega})$ (Equation (10)) by substituting $p(L \mid \boldsymbol{d})=p(F \mid \boldsymbol{d}) d F / d L=p(F \mid \boldsymbol{d}) / 4 \pi D^{2}$ and $d L=4 \pi D^{2} d F$, giving

$$
0.95=\int d D \int d \hat{\Omega} \int_{0}^{F\left(D, L_{95}\right)} d F p(F \mid d, \hat{\Omega}) p(D, \hat{\Omega}) .
$$

In practice, Equation (15) is somewhat easier to evaluate when rearranged as

$$
0.95=\int d \hat{\Omega} p(\hat{\Omega}) \int d D p(D \mid \hat{\Omega}) \int_{0}^{F\left(D, L_{95}\right)} d F p(F \mid \boldsymbol{d}, \hat{\Omega}) .
$$

\section{ORCID iDs}

Thomas A. Callister (ib https://orcid.org/0000-00019892-177X

Jayce Dowell (1D https://orcid.org/0000-0003-1407-0141

Namir E. Kassim (i) https://orcid.org/0000-0001-8035-4906

Danny C. Price (1) https://orcid.org/0000-0003-2783-1608

Frank K. Schinzel (i) https://orcid.org/0000-0001-6672-128X

\section{References}

Abbott, B. P., Abbott, R., Abbott, T. D., et al. 2016, PhRvL, 116, 061102 Abbott, B. P., Abbott, R., Abbott, T. D., et al. 2017a, PhRvL, 119, 161101 Abbott, B. P., Abbott, R., Abbott, T. D., et al. 2017b, ApJL, 848, L12 Abbott, B. P., Abbott, R., Abbott, T. D., et al. 2017c, Natur, 551, 85 Abbott, B. P., Abbott, R., Abbott, T. D., et al. 2017d, PhRvL, 118, 221101 Abbott, B. P., Abbott, R., Abbott, T. D., et al. 2017e, ApJL, 848, L13 Abbott, B. P., Abbott, R., Abbott, T. D., et al. 2018a, LRR, 21, 3 Abbott, B. P., Abbott, R., Abbott, T. D., et al. 2018b, PhRvL, 121, 161101 Abbott, B. P., Abbott, R., Abbott, T. D., et al. 2019, ApJ, 875, 161 Anderson, M. M., Hallinan, G., Eastwood, M. W., et al. 2018, ApJ, 864, 22 Baars, J. W. M., Genzel, R., Pauliny-Toth, I. I. K., \& Witzel, A. 1977, A\&A, 61, 99

Bannister, K. W., Murphy, T., Gaensler, B. M., \& Reynolds, J. E. 2012, ApJ, 757,38

Berger, E. 2014, ARA\&A, 52, 43

Callister, T., Dowell, J., Kanner, J., et al. 2017, GCN, 21680, 1

Cantiello, M., Jensen, J. B., Blakeslee, J. P., et al. 2018, ApJL, 854, L31

Ceplecha, Z., Borovička, J., Elford, W. G., et al. 1998, SSRv, 84, 327

Chu, Q., Howell, E. J., Rowlinson, A., et al. 2016, MNRAS, 459, 121

Connaughton, V., Burns, E., Goldstein, A., et al. 2016, ApJL, 826, L6

Connaughton, V., Burns, E., Goldstein, A., et al. 2018, ApJL, 853, L9

Cordes, J. M., \& Lazio, T. J. W. 2002, arXiv:astro-ph/0207156

Cordes, J. M., \& Lazio, T. J. W. 2003, arXiv:astro-ph/0301598

Cordes, J. M., Wharton, R. S., Spitler, L. G., Chatterjee, S., \& Wasserman, I. 2016, arXiv: 1605.05890

Coulter, D. A., Foley, R. J., Kilpatrick, C. D., et al. 2017, Sci, 358, 1556 
de Mink, S. E., \& King, A. 2017, ApJL, 839, L7

Drout, M. R., Piro, A. L., Shappee, B. J., et al. 2017, Sci, 358, 1570

Eastwood, M. W. 2016, TTCal, Zenodo, doi:10.5281/zenodo.1049160

Eastwood, M. W., Anderson, M. M., Monroe, R. M., et al. 2018, ApJ, 156, 32

Fraschetti, F. 2018, JCAP, 2018, 054

Goldstein, A., Veres, P., Burns, E., et al. 2017, ApJL, 848, L14

Greiner, J., Burgess, J. M., Savchenko, V., \& Yu, H.-F. 2016, ApJL, 827, L38

Hallinan, G., Corsi, A., Mooley, K. P., et al. 2017, Sci, 358, 1579

Hansen, B. M. S., \& Lyutikov, M. 2001, MNRAS, 322, 695

Helmboldt, J. F., Ellingson, S. W., Hartman, J. M., et al. 2014, RaSc, 49, 157

Hicks, B. C., Paravastu-Dalal, N., Stewart, K. P., et al. 2012, PASP, 124, 1090

Hotokezaka, K., Nakar, E., Gottlieb, O., et al. 2018, arXiv:1806.10596

Inoue, S. 2004, MNRAS, 348, 999

Ioka, K. 2003, ApJL, 598, L79

Kaplan, D., Brown, I., Sokolowski, M., et al. 2017, GCN, 21927, 1

Kaplan, D. L., Murphy, T., Rowlinson, A., et al. 2016, PASA, 33, e050

Kaplan, D. L., Rowlinson, A., Bannister, K. W., et al. 2015, ApJL, 814, L25

Kocz, J., Greenhill, L. J., Barsdell, B. R., et al. 2015, JAI, 4, 1550003

Lai, D. 2012, ApJL, 757, 1

Liebling, S. L., \& Palenzuela, C. 2016, PhRvD, 94, 064046

LIGO Scientific Collaboration \& Virgo Collaboration 2018, LIGO/Virgo Public Alerts User Guide, https://emfollow.docs.ligo.org/userguide/index.html

Liu, T., Romero, G. E., Liu, M.-L., \& Li, A. 2016, ApJ, 826, 82

Loeb, A. 2016, ApJL, 819, L21

Lyutikov, M. 2019, MNRAS, 483, 2766

Lyutikov, M. 2016, arXiv:1602.07352
Lyutikov, M. 2013, ApJ, 768, 63

Metzger, B. D., \& Zivancev, C. 2016, MNRAS, 461, 4435

Mooley, K. P., Frail, D. A., Dobie, D., et al. 2018, ApJL, 868, L11

Nakar, E., Gottlieb, O., Piran, T., Kasliwal, M. M., \& Hallinan, G. 2018, ApJ, 867,18

Obenberger, K. S., Hartman, J. M., Taylor, G. B., et al. 2014, ApJ, 785, 27

Offringa, A. R., McKinley, B., Hurley-Walker, N., et al. 2014, MNRAS, 444, 606

Perley, R. A., \& Butler, B. J. 2017, ApJS, 230, 7

Perna, R., Lazzati, D., \& Giacomazzo, B. 2016, ApJL, 821, L18

Pshirkov, M. S., \& Postnov, K. A. 2010, Ap\&SS, 330, 13

Raithel, C. A., Özel, F., \& Psaltis, D. 2018, ApJL, 857, L23

Ravi, V., \& Lasky, P. D. 2014, MNRAS, 441, 2433

Rodriguez, C. L., Amaro-Seoane, P., Chatterjee, S., et al. 2018, PhRvD, 98, 123005

Rowlinson, A., \& Anderson, G. E. 2019, arXiv:1905.02509

Savchenko, V., Ferrigno, C., Mereghetti, S., et al. 2016, ApJL, 820, L36

Smartt, S. J., Chen, T.-W., Jerkstrand, A., et al. 2017, Natur, 551, 75

Totani, T. 2013, PASJ, 65, L12

Troja, E., Piro, L., van Eerten, H., et al. 2017, Natur, 551, 71

Usov, V. V., \& Katz, J. I. 2000, A\&A, 364, 655

Wang, J.-S., Peng, F.-K., Wu, K., \& Dai, Z.-G. 2018, ApJ, 868, 19

Wang, J.-S., Yang, Y.-P., Wu, X.-F., Dai, Z.-G., \& Wang, F.-Y. 2016, ApJL, 822, L7

Yancey, C. C., Bear, B. E., Akukwe, B., et al. 2015, ApJ, 812, 168

Zhang, B. 2016, ApJL, 827, L31 